\title{
Éditorial
}

\section{Un très intéressant congrès, mais (une panne de propositions d'articles)...}

Depuis plusieurs années, notre société se félicite de l'excellente tenue de ses manifestations et congrès. Le dernier congrès tenu à Angers, du 16 au 19 juin, n'a pas dérogé à cette évolution, loin s'en faut... Tant les présentations orales que les posters ont été salués pour la rigueur scientifique des uns, l'effort pédagogique des autres, mais aussi la prise en compte par tous des problèmes pratiques et concrets qui jalonnent la radioprotection quotidienne.

Ces avancées risquent toutefois de rester orphelines si la diffusion qu'elles méritent n'est pas assurée. J'ai, bien entendu, grand plaisir à saluer l'initiative heureuse de notre secrétaire général, qui s'est d'ores et déjà attaché à mettre ces travaux en ligne sur le site de la SFRP. Néanmoins, force est de reconnaître que seules les publications offrent aux travaux scientifiques l'aura qu'ils méritent. Et cela est particulièrement vrai pour nos collègues étrangers à l'heure où le «benchmarking » devient incontournable...

Aussi, le président du Comité de rédaction a choisi de dédier cet éditorial à une exhortation à la soumission de projets d'articles relatifs aux travaux présentés à Angers. Pour la plupart des auteurs, l'effort à fournir est modeste et je compte sur eux pour nous envoyer un projet qui, une fois publié dans une revue à comité de lecture, pourra être cité et accessible via les principaux moteurs de recherches.

J'en profite pour remercier chaleureusement tous ceux qui ont déjà fait cette démarche et qui, je l'espère, ne le regrettent pas...

Nous avons d'excellents évaluateurs, rigoureux et exigeants, qui contribuent à garantir le niveau scientifique auquel notre revue aspire ; vos travaux gagnent à être connus et reconnus, nous nous y employons.

J'aimerais ainsi pouvoir compter sur une nouvelle « Révélation »-après celle proposée par la Tenture de l'Apocalypse - révélation qui consisterait à citer désormais ce congrès en référence, en raison du grand nombre d'articles qu'il a générés !...

DOI: $10.1051 /$ radiopro/2009017 
ÉDITORIAL

Si vous allez sur Internet vous lirez presque la même chose en 2004 (Radioprotection, volume $39, \mathrm{~N}^{\circ} 1$ ), et je vous laisse réfléchir à la dernière phrase...

À vos plumes

H. Métivier

Président du Comité de rédaction

PS: Au moment de la rédaction de cet éditorial, nous avons eu le plaisir de recevoir le numéro spécial « ECORAD 2008 » (Radioprotection, volume 44, N 5, 2009), actes du congrès « Radioecology and Environmental Radioactivity », Bergen, juin 2008, que nous devons à la fidélité de notre ami Jean-Claude Barescut et à l'aide de l'IRSN ; le Comité de rédaction les remercie vivement. Cet excellent numéro spécial, comme tous les numéros spéciaux, est consultable sur le site Internet de la revue dès parution. 